\title{
Honokiol regulates endoplasmic reticulum stress by promoting the activation of the sirtuin 1-mediated protein kinase $B$ pathway and ameliorates high glucose/ high fat-induced dysfunction in human umbilical vein endothelial cells
}

\author{
Hong $\mathrm{Ye}^{1) *}$ and Ying Meng ${ }^{2) *}$ \\ 1) Department of Cardiovascular Medicine, Anhui Chest Hospital, Hefei City, Anhui Province, 230000, China \\ 2) Department of Cardiology, Hefei Binhu Hospital, Hefei City, Anhui Province, 230011, China
}

\begin{abstract}
Honokiol plays an important role in anti-oxidation, but its role in diabetic vascular complications is unclear. In this study, the effects of honokiol in high glucose/high fat (HG/HF)-induced human umbilical vein endothelial cells (HUVECs) were explored. After pre-treatment with honokiol, the cells were transferred to an HG/HF medium, and cell viability and apoptosis were respectively measured by methyl tetrazolium and flow cytometry. Moreover, the contents of reactive oxygen species (ROS), malondialdehyde (MDA), and superoxide dismutase (SOD) were measured. The expressions of C/EBP homologous protein (CHOP), glucose-regulated protein 78 (GRP78), phosphorylated-protein kinase RNA-like endoplasmic reticulum kinase (p-PERK), phosphorylated-inositol requiring enzyme-1 $\alpha$ (p-IRE1 $\alpha$ ), cleaved caspase-3 and SIRT1 were determined by Western blot or quantitative reverse transcription PCR, respectively. Finally, the viability, apoptosis, and the contents of ROS, MDA, and SOD, as well as the expressions of CHOP, GRP78, p-PERK, p-IRE1 $\alpha$, cleaved caspase-3, Akt, pAkt, and SIRT1 in the cells transfected with small interfering RNA SIRT1 (siSIRT1) were detected by the previously mentioned methods. Honokiol reversed the effect of HG/HF on promoting cell apoptosis, ROS and MDA contents, and the expressions of CHOP, GRP78, p-PERK, p-IRE1 $\alpha$ and cleaved caspase-3, and also reversed the inhibitory effect of HG/HF on cell viability, SOD content and SIRT1 expression. However, siSIRT1 reversed the above effects caused by honokiol. Honokiol activated SIRT1 promoter. SIRT1 interacted with Akt, consequently promoting the activity of Akt. Therefore, honokiol activates the Akt pathway by regulating SIRT1 expression to regulate endoplasmic reticulum stress, promotes cell viability and inhibits the apoptosis of HG/HF-induced HUVECs.
\end{abstract}

Key words: Honokiol, Human umbilical vein endothelial cells (HUVECs), Endoplasmic reticulum stress, Akt, Apoptosis

DIABETES MELLITUS is a chronic disease with high blood glucose resulted from insufficient insulin secretion or insulin resistance [1]. In recent years, the incidence of diabetes mellitus has been steadily increasing [2]. Studies have shown that with the aggravation of insulin resistance and the extension of the course of disease in diabetic patients, diabetic complications, such as microvascular complications, macrovascular complications and neurological complications, will affect systemic vital

Submitted Nov. 25, 2020; Accepted Mar. 10, 2021 as EJ20-0747 Released online in J-STAGE as advance publication Apr. 29, 2021 Correspondence to: Hong Ye, Department of Cardiovascular Medicine, Anhui Chest Hospital, No. 397 Jixi Road, Shushan District, Hefei City, Anhui Province, 230000, China.

E-mail: yehong_yh1@163.com

*These authors contributed equally to this work. organs, and that vascular complications are an important cause of death to diabetic patients [3-6]. Therefore, the prevention and treatment of diabetic vascular complications has become one of the hotspots in the field of diabetes-related research.

Endoplasmic Reticulum (ER) stress is an evolutionarily highly conserved cellular response mechanism and is one of the pathways through which cells undergo apoptosis. Hypoxia and oxidative stress responses can trigger the development of ER stress in endothelial cells and cancers [7-11]. Studies have shown that ER stress is involved in the development and progression of a variety of diseases [12]. Related studies have found that ER stress plays an important role in diabetes-related cardiovascular disorders and endothelial dysfunctions, and ER stress has the potential to serve as a targeted therapy for 
diabetic atherosclerosis [13, 14]. Specifically, in studies related to ER stress and atherosclerosis, salicylate was found to inhibit inflammation and ER stress by regulating the expressions of Heme oxygenase 1 (HO-1) and sirtuin 1 (SIRT1), which in turn improved atherosclerotic response [15]. SIRT1, which is a nicotinamide adenosine dinucleotide (NAD)+-dependent deacetylase, has been found to promote cardiomyocyte survival by attenuating the ER stress response; in addition, multiple studies demonstrated that SIRT1 is involved in regulating ER stress responses [16-19].

Honokiol is a plant active component with low molecular weight and high safety [20], exerting an important role in liver protection, anti-oxidation and glucose and lipid metabolism [21-23]. In addition, honokiol can reduce visceral adipose tissue weight and adipocyte size in mice, and prevent the development of insulin resistance. Moreover, honokiol could stimulate glucose transporter 4 translocation to the myocyte surface through the PI3K-Akt signaling pathway [24, 25]. Honokiol improved myocardial ischemia/reperfusion injury in diabetic rats by mediating SIRT1-Nrf2 signaling pathway and reducing oxidative stress [26]. However, it is unclear whether honokiol can improve high glucose/high fat (HG/HF)-induced human umbilical vein endothelial cells (HUVECs) function through SIRT1 regulation of ER stress.

Based on the above research background, we explored the mechanism of honokiol on the HG/HF-induced HUVECs. The results of this experiment provide a reliable theoretical basis for the treatment and prevention of diabetic vascular complications.

\section{Methods and Materials}

\section{Cells culture}

HUVECs (PCS-100-013) were purchased from American Type Culture Collection (ATCC; Maryland, USA), and the cells were cultured in Vascular Cell Basal Medium (PCS-100-030, ATCC, Maryland, USA) according to the manufacturer's instructions. The components contained in the Endothelial Cell Growth Kit-VEGF (PCS-100-041, ATCC, Maryland, USA) were added to the medium. Afterwards, the seeded cells were cultured in an incubator (BC-J80, Boxun, Shanghai, China) with $5 \% \mathrm{CO}_{2}$ at $37^{\circ} \mathrm{C}$, and the medium was changed every 2 days.

\section{Cells transfection}

The specific small interfering RNA (siRNA) targeting SIRT1 (siSIRT1 target sequence: 5'-GTCGCTTTAAAAT AAGTTTCTCT-3') and siSIRT1 negative control (siNC; 5'-UUCUCCGAACGUGUCACGUTT-3') were purchased from GenePharma Company (Shanghai, China). The HUVECs at the logarithmic growth phase were collected, washed with Phosphate-Buffered Saline (PBS; 10010031, Thermo Fisher, Massachusetts, USA), and then the cell concentration was adjusted to approximately $1 \times 10^{5}$ cells $/ \mathrm{mL}$. Next, the cells were transferred to a 6-well plate for continued culture. When the cells confluence reached approximately $80 \%$, siSIRT1 and siNC were transfected into HUVECs for $48 \mathrm{~h}$, respectively, according to the operating instructions of Lipofectamine $^{\mathrm{TM}} 2000$ Transfection Reagent (11668019, Thermo Fisher, Massachusetts, USA).

\section{Cells treatment}

To determine the treatment concentration of honokiol (H4914, Sigma-Aldrich, Missouri, USA), honokiol was dissolved in dimethyl sulfoxide (DMSO; D2650, SigmaAldrich, Missouri, USA) mother liquor, according to the previous experimental method $[27,28]$. Then the cells were collected and divided into the following five groups: (1) Control group: HUVECs were incubated in medium for $2 \mathrm{~h}$ and then cultured in culture medium containing normal amount $(5 \mathrm{mmol} / \mathrm{L})$ of glucose (G8270, Sigma-Aldrich, Missouri, USA) for $24 \mathrm{~h}$; (2) HG/HF group: HUVECs were cultured in medium containing high glucose $(25 \mathrm{mmol} / \mathrm{L})$ and high saturated free fatty acid palmitate $(500 \mu \mathrm{mol} / \mathrm{L}, 1170460001$, SigmaAldrich, Missouri, USA) for $24 \mathrm{~h}$ after incubation in medium for $2 \mathrm{~h}$; (3) $\mathrm{HG} / \mathrm{HF}+$ Honokiol $(5 \mu \mathrm{mol} / \mathrm{L})$ group: HUVECs were pretreated with medium containing $5 \mu \mathrm{mol} / \mathrm{L}$ Honokiol for $2 \mathrm{~h}$, and then transferred to medium containing HG/HF cultured for $24 \mathrm{~h}$; (4) HG/HF + Honokiol $(20 \mu \mathrm{mol} / \mathrm{L})$ group: HUVECs were pretreated with medium containing $20 \mu \mathrm{mol} / \mathrm{L}$ Honokiol for $2 \mathrm{~h}$, and then transferred to medium containing $\mathrm{HG} / \mathrm{HF}$ cultured for $24 \mathrm{~h}$; (5) HG/HF + Honokiol $(80 \mu \mathrm{mol} / \mathrm{L})$ group: HUVECs were pretreated with medium containing $80 \mu \mathrm{mol} / \mathrm{L}$ Honokiol for $2 \mathrm{~h}$, and then transferred to medium containing HG/HF cultured for $24 \mathrm{~h}$.

The transfected HUVECs were collected and cultured in normal medium or $\mathrm{HG} / \mathrm{HF}$ medium for $24 \mathrm{~h}$, or the transfected cells were pretreated with $80 \mu \mathrm{mol} / \mathrm{L}$ Honokiol for $2 \mathrm{~h}$ and then transferred to medium containing $\mathrm{HG} / \mathrm{HF}$ cultured for $24 \mathrm{~h}$.

\section{Detection of cell viability}

The viability of the HUVECs was determined by Methyl tetrazolium (MTT) cell viability Assay Kit (V13154, Thermo Fisher, Massachusetts, USA). The cells were collected and transferred to 96-well plates after adjusting cell concentration $\left(5 \times 10^{3}\right.$ cells/well). After the cells in each group were appropriately treated, an appropriate amount $(10 \mu \mathrm{L}) 12$-mM MTT stock solution 
was added to each well according to the operating instructions, and then the cells were incubated at $37^{\circ} \mathrm{C}$ for $4 \mathrm{~h}$. Next, $100 \mu \mathrm{L}$ SDS-HCl solution was added to each well and the cells were incubated for $4 \mathrm{~h}$. Finally, the absorbance of each sample well at $570 \mathrm{~nm}$ was detected with a microplate reader (SpectraMax iD3, Molecular Devices, Calif, USA).

\section{Detection of cell apoptosis}

Cell apoptosis was detected by Annexin V-FITC/ Propidium Iodide Apoptosis Detection Kit (BMS500FI-100, Thermo Fisher, Massachusetts, USA). According to the protocol, the treated HUVECs in each group were collected, washed with PBS, and then resuspended in Binding Buffer to adjust the cell concentration to $5 \times 10^{5}$ cells $/ \mathrm{mL}$. Subsequently, $5 \mu \mathrm{L}$ Annexin V-FITC was added to the cell suspension, and treated for $10 \mathrm{~min}$ at room temperature. Then the cells washed with Binding Buffer, and resuspended in Binding Buffer. Finally, the cells were treated with the $10 \mu \mathrm{L}$ Propidium Iodide and then analyzed by flow cytometry (CytoFLEX, Beckman Coulter, Calif, USA).

\section{Detection of intracellular reactive oxygen species (ROS)}

Intracellular ROS level was detected by ROS Detection Kit (BB-47053-1, BestBio, Shanghai, China). According to the kit instruction, the treated HUVECs in each group were collected, and transferred to 6-well plates. DCFHDA was first diluted with serum-free medium at a ratio of 1:1,000, and then added into each well to culture the cells in an incubator for $20 \mathrm{~min}$. Next, the cells were washed again with serum-free medium, and the fluorescence intensity of the samples was measured with a fluorospectro photometer (83061-01, ColeParmer, Illinois, USA). The ROS level was analyzed based on the fluorescence intensity of the samples.

\section{Determination of malondialdehyde (MDA) and superoxide dismutase (SOD) contents}

Cell MDA content was measured by Lipid Peroxidation MDA Assay Kit (S0131S, Beyotime, Shanghai, China). After collecting the treated HUVECs in each group, the cells were lysed with cell lysate (P0013, Beyotime, Shanghai, China), and then centrifuged (Avanti J-15R, Beckman Coulter, Calif, UAS) at 10,000 $\times g$ for $10 \mathrm{~min}$ before the supernatant had been taken for subsequent measurements. Subsequently, the protein concentration was measured with a BCA Protein Assay Kit (P0009, Beyotime, Shanghai, China). Then the TBA stock solution and MDA detection working solution as well as the diluted standards were prepared according to the manufacturer's instructions. Finally, the absorbance of the samples was measured with a microplate reader at $532 \mathrm{~nm}$ and the content of MDA was calculated.

The SOD levels of HUVECs were measured by a SOD Assay Kit (ml076328, Enzyme-linked Biotechnology Co., Ltd., Shanghai, China). The treated cells in each group were collected into centrifuge tubes, and an appropriate amount of extract was added to the centrifuge tubes. After the cells were broken by ultrasonic waves, and then centrifuged at $8,000 \times \mathrm{g}$ for $10 \mathrm{~min}$ at $4{ }^{\circ} \mathrm{C}$. Afterwards, the supernatant was taken to detect the SOD content of the samples.

\section{Quantitative reverse transcription PCR (RT-qPCR)}

The treated HUVECs in each group were collected into centrifuge tubes, and washed with PBS after centrifugation, and then mRNAs were extracted from the cells by mRNA preparation Kit (MRN70, Sigma-Aldrich, Missouri, USA). Afterwards, the purity of mRNAs was determined by UV spectrophotometer (DU-720, Beckman Coulter, Calif, USA), and the integrity of the mRNAs was examined by agarose gel (ST004Q, Beyotime, Shanghai, China) electrophoresis. The cDNA was synthesized using a cDNA Reverse-transcription Kit (4374966, Thermo Fisher, Massachusetts, USA), followed by PCR amplification. In the experiment, the expression of SIRT1 was detected by a RT-qPCR system (CFX96 Touch Real-Time PCR, Bio-Rad, Calif, USA), with SYBR ${ }^{\mathrm{TM}}$ Green Master Mix (A25742, Thermo Fisher, Massachusetts, USA). The primer synthesis sequences were as follows: SIRT1 (F: 5'-TAGCCTTGTC AGATAAGGAAGGA-3'，R: 5'-ACAGCTTCACAGTC AACTTTGT-3'), GAPDH (F: 5'-GGAGCGAGATCCCT CCAAAAT-3', R: 5'-GGCTGTTGTCATACTTCTCATG G-3'). In this experiment, GAPDH was a reference gene and the results were analyzed by the $2^{-\Delta \Delta \mathrm{ct}}$ method.

\section{Western blot}

The HUVECs in each group were collected and digested with Trypsin-EDTA Solution (C0201, Beyotime, Shanghai, China), and the cell concentration was adjusted to $5 \times 10^{5}$ cells $/ \mathrm{mL}$. After centrifugation, the supernatant was discarded and the cells were washed with precooled PBS, and then an appropriate amount of RIPA lysis and Extraction Buffer (89900, Thermo Fisher, Massachusetts, USA) was added to the centrifuge tube to extract total protein from the cells. Then the total protein concentration was measured with a BCA Protein Assay Kit (P0009, Beyotime, Shanghai, China). Polyacrylamide gel was prepared by SDS-PAGE Gel Kit (P1200, Solarbio, Beijing, China), and then equal amounts $(25 \mu \mathrm{L})$ of protein samples were added to the sample wells of the gel, followed by electrophoresis. At the end of electrophoresis experiments, the gel was removed, and 
the protein was transferred to a PVDF membrane (YA1701, Solarbio, Beijing, China), which was blocked with Western Blocking Buffer (SW3010, Solarbio, Beijing, China) for $60 \mathrm{~min}$ at room temperature. After that, the membranes were washed with Tris Buffered saline Tween (TBST; T1085, Solarbio, Beijing, China) 3 times for 3 min each time. PVDF membrane was then treated overnight at $4{ }^{\circ} \mathrm{C}$ in primary antibodies. After that, the PVDF membrane was washed with TBST solution 3 times for $3 \mathrm{~min}$ each time, and then the membrane was incubated in the secondary antibody for $1 \mathrm{~h}$ at room temperature. Afterwards, the membranes were washed with TBST, and then the membranes were completely immersed in ECL Plus working solution (PE0010, Solarbio, Beijing, China) for $3 \mathrm{~min}$ at room temperature in the dark. Finally, the gel was analyzed on a gel image processing system (FluorChem FC3, Alpha Innotech, Calif, USA). All the information and sources of antibodies were shown in Table 1.

\section{Co-immunoprecipitation (Co-IP) assay}

Co-IP assay was performed to identify the direct interrelation between SIRT1 and Akt on HUVECs. Firstly, immunoprecipitation (IP) was performed on HUVECs using the Abcam immunoprecipitation kit (ab206996), following the manufacturer's instructions. Briefly, nondenaturing lysis buffer was used to collect cell lysate. A small amount of cell lysate was used for Western blot analysis to detect the expressions of SIRT1 and Akt, and while other cell lysate were used for IP. Then, $300 \mu \mathrm{g}$ of cell lysate was incubated overnight with $3 \mu \mathrm{g} / \mathrm{mL}$ of either control mouse IgG (sc-2025, Santa Cruz,) or SIRT1 mouse monoclonal antibody (ab110304, Abcam). Antibody- bound proteins were captured using protein $\mathrm{A} / \mathrm{G}$ sepharose beads, eluted, and analyzed via Western blot.

\section{Dual luciferase reporter analysis}

SIRT1 promoter sequences were amplified by PCR using upstream and downstream primers of SIRT1 promoter: F: 5'-CCGCTCGAGCTACACGCTCGCCACAA A-3'; R: 5'-CCCAAGCTT CCGCCATCTTCCAACTG C-3'. The human SIRT1 promoter sequences were located into the XhoI/HindIII-digested pGL3-Basic reporter vector to construct the pGL3-Basic-SIRT1. Cells were subjected to transfection of pGL3-Basic-SIRT1 and internal reference PRL-TK plasmid, for $24 \mathrm{~h}$ and treated with honokiol at 5-80 $\mu \mathrm{mol} / \mathrm{L}$. Dual-luciferase reporter assay system (E1910, Promega, Madison, WI, USA) was applied for analysis of the luciferase activities. The promoter activities were calculated by normalization of the ratio between the activities of firefly luciferase and Renilla luciferase.

\section{Statistical analysis}

There are three biological replicates and three technical replicates for each biological replicate. Measurement data were described by mean \pm standard deviation, and multiple groups were compared using One-way ANOVA. All statistical analyses were implemented using Graphpad 8.0 software. $P<0.05$ was considered as statistically significant.

\section{Results}

\section{Honokiol reversed the inhibitory effect of $\mathrm{HG} / \mathrm{HF}$ on} cell viability and the promoting effect on apoptosis

From Fig. 1A, it can be observed that HG/HF treatment prominently inhibited cell viability compared with the Control group $(p<0.001)$, however, after the cell pretreatment with honokiol at $5 \mu \mathrm{mol} / \mathrm{L}, 20 \mu \mathrm{mol} / \mathrm{L}$ and $80 \mu \mathrm{mol} / \mathrm{L}$, honokiol notably reversed the inhibitory

Table 1 All antibodies information and sources in this study

\begin{tabular}{|c|c|c|c|c|}
\hline ID & Catalog number & Company (country) & Molecular weight & Dilution ratio \\
\hline CHOP & ab11419 & Abcam (Cambridge, UK) & $31 \mathrm{kDa}$ & $1 / 1,000$ \\
\hline GRP78 & ab21685 & Abcam (Cambridge, UK) & $75 \mathrm{kDa}$ & $1 / 1,000$ \\
\hline p-PERK & DF7576 & Affinity Biosciences (Massachusetts, USA) & $125 \mathrm{kDa}$ & $1 / 1,000$ \\
\hline $\mathrm{p}-\mathrm{IRE} 1 \alpha$ & ab48187 & Abcam (Cambridge, UK) & $110 \mathrm{kDa}$ & $1 / 1,000$ \\
\hline Cleaved caspase- 3 & ab2302 & Abcam (Cambridge, UK) & $17 \mathrm{kDa}$ & $1 / 500$ \\
\hline SIRT1 & ab110304 & Abcam (Cambridge, UK) & $81 \mathrm{kDa}$ & $1 / 1,000$ \\
\hline p-Akt & ab38449 & Abcam (Cambridge, UK) & $56 \mathrm{kDa}$ & $1 / 1,000$ \\
\hline Akt & ab8805 & Abcam (Cambridge, UK) & $55 \mathrm{kDa}$ & $1 / 500$ \\
\hline GAPDH & ab8245 & Abcam (Cambridge, UK) & $36 \mathrm{kDa}$ & $1 / 1,000$ \\
\hline Mouse IgG & ab205719 & Abcam (Cambridge, UK) & & $1 / 5,000$ \\
\hline Rabbit IgG & ab205718 & Abcam (Cambridge, UK) & & $1 / 5,000$ \\
\hline
\end{tabular}


effect of $\mathrm{HG} / \mathrm{HF}$ on cell viability as compared with the HG/HF group $(p<0.05)$. By detecting the apoptosis of HUVECs using flow cytometry, we found that $\mathrm{HG} / \mathrm{HF}$ significantly promoted apoptosis compared with the Control group $(p<0.001)$, whereas after the cell pretreatment with honokiol, honokiol reduced the effect of $\mathrm{HG} / \mathrm{HF}$ on promoting apoptosis as compared with the $\mathrm{HG} / \mathrm{HF}$ group (Fig. 1B-C, $p<0.01$ ).

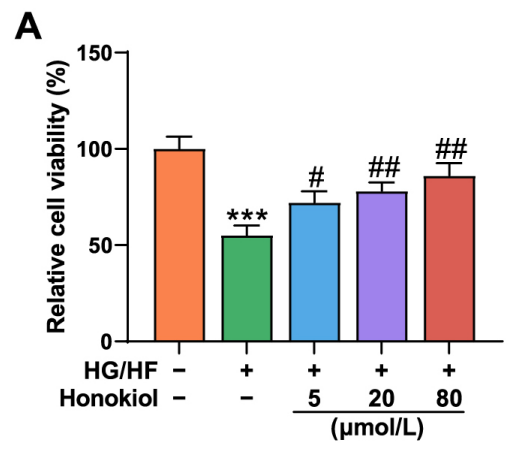

\section{Honokiol reversed the effect of $\mathrm{HG} / \mathrm{HF}$ on promoting ROS and MDA contents as well as the inhibitory effect on SOD contents in HUVECs}

As shown in Fig. 1D and 1E, the ROS level was based on fluorescence intensity of the samples, and MDA content in HUVECs was detected. The results showed that HG/HF obviously increased the contents of ROS and MDA in the cells as compared with the Control group ( $p$ $<0.001$ ), whereas honokiol clearly inhibited the effect of $\mathrm{HG} / \mathrm{HF}$ on increasing the contents of ROS and MDA in

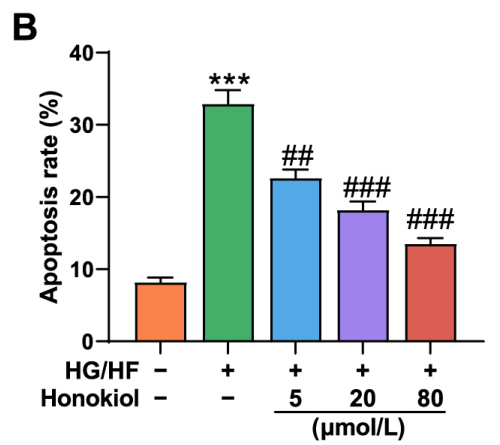

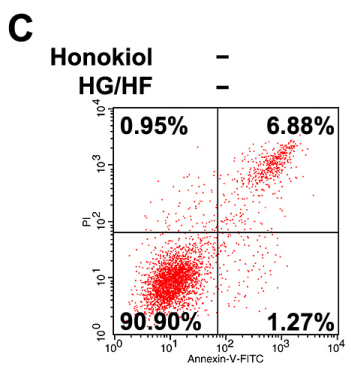
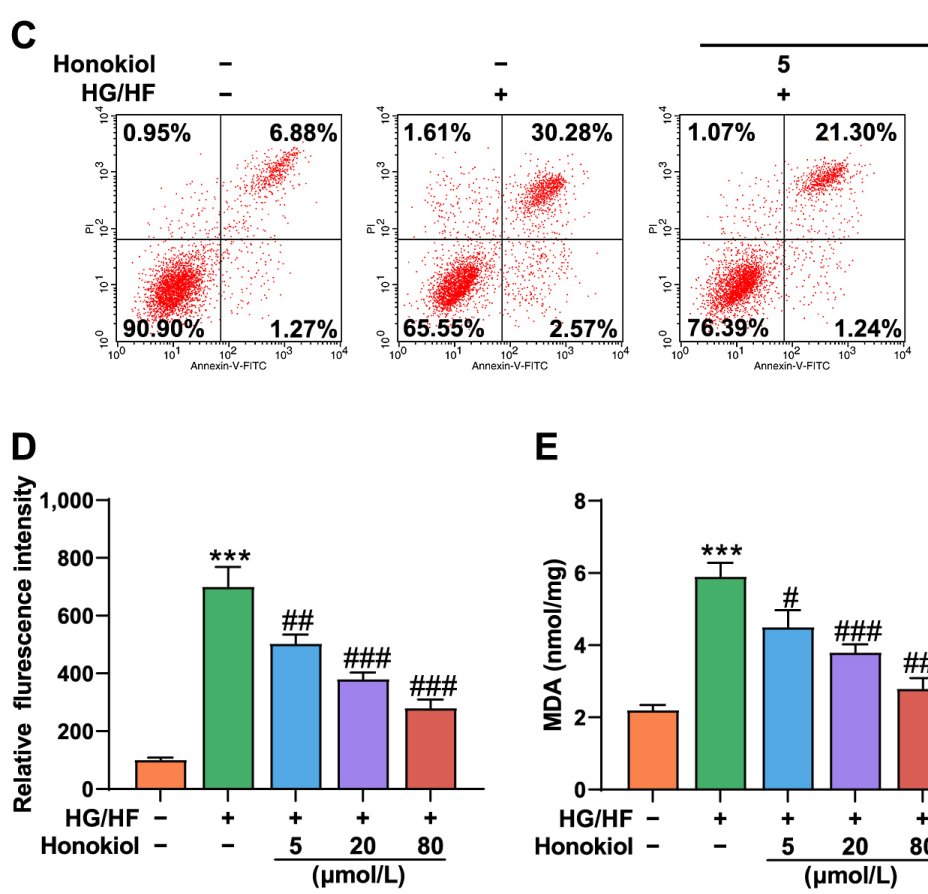

E

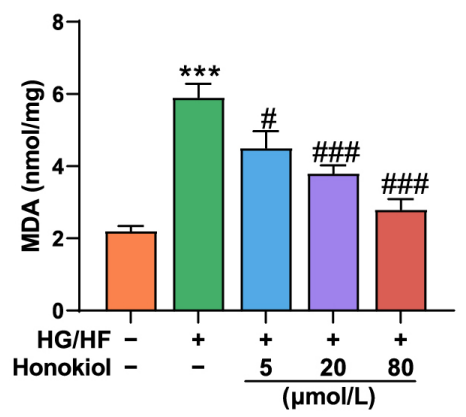

$\mathbf{F}$

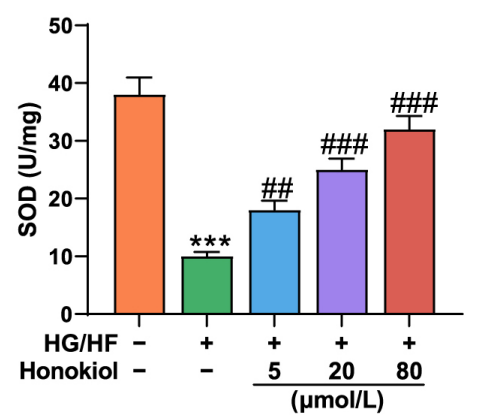

Fig. 1 Honokiol reversed the inhibitory effect of HG/HF on cell viability and SOD content; also reversed the effect on promoting apoptosis and the contents of ROS and MDA

(A) The viability of HG/HF-induced HUVECs after pre-treatment with different concentrations $(5,20$ and $80 \mu \mathrm{mol} / \mathrm{L})$ of honokiol was examined by MTT. (B-C) The apoptosis of HG/HF-induced HUVECs after pre-treatment with different concentrations $(0,5$, 20 and $80 \mu \mathrm{mol} / \mathrm{L}$ ) of honokiol was examined by flow cytometry. (D) The ROS contents of HG/HF-induced HUVECs after pretreatment with different concentrations $(5,20$ and $80 \mu \mathrm{mol} / \mathrm{L}$ ) of honokiol were examined. (E) The MDA content of HG/HFinduced HUVECs after pre-treatment with different concentrations $(5,20$ and $80 \mu \mathrm{mol} / \mathrm{L})$ of honokiol was examined. (F) The SOD content of HG/HF-induced HUVECs after pre-treatment with different concentrations $(5,20$ and $80 \mu \mathrm{mol} / \mathrm{L})$ of honokiol was examined. ${ }^{* * *} p<0.001$ vs. Control; ${ }^{\#} p<0.05,{ }^{\#} p<0.01,{ }^{\# \#} p<0.001$ vs. HG/HF. (HG/HF, high glucose/high fat; HUVECs, human umbilical vein endothelial cells; MMT, Methyl tetrazolium; ROS, reactive oxygen species; MDA, malondialdehyde; SOD, superoxide dismutase) 
HUVECs when compared with the HG/HF group ( $p<$ $0.05)$. In addition, we found that $\mathrm{HG} / \mathrm{HF}$ inhibited the contents of SOD in comparison with the Control group $(p<0.001)$, but honokiol markedly promoted the contents of SOD in HUVECs compared with the HG/HF group (Fig. 1F, $p<0.01$ ).

\section{Honokiol reversed the effect of $\mathrm{HG} / \mathrm{HF}$ on promoting the expressions of ER stress markers and cleaved caspase-3 as well as the inhibitory effect on SIRT1 expression in HUVECs}

As shown in Fig. 2A and 2B, Western blot data showed that $\mathrm{HG} / \mathrm{HF}$ promoted the expressions of ER stress markers, including $\mathrm{C} / \mathrm{EBP}$ homologous protein (CHOP), glucose-regulated protein 78 (GRP78), phosphorylated-protein kinase RNA-like endoplasmic reticulum kinase (p-PERK) and phosphorylated-inositol requiring enzyme-1 $\alpha$ (p-IRE1 $\alpha$ ), and also promoted cleaved caspase-3 expression, as compared with the Control group $(p<0.001)$. Honokiol partially counteracted the effect of $\mathrm{HG} / \mathrm{HF}$ on promoting the expressions of ER stress markers and cleaved caspase- 3 in the cells ( $p<$ $0.05)$. In addition, the results of Western blot and RTPCR experiments indicated that $\mathrm{HG} / \mathrm{HF}$ inhibited the expression of SIRT1 in comparison with the Control group $(p<0.001)$, whereas honokiol partially reversed the inhibitory effect of $\mathrm{HG} / \mathrm{HF}$ on SIRT1expression in the HUVECs, as compared with the HG/HF group (Fig. $2 \mathrm{C}-\mathrm{E}, p<0.05$ ). As shown in Fig. $2 \mathrm{~F}$, relative luciferase activity of SIRT1 promoter was decreased as honokiol concentration increased from 5 to $80 \mu \mathrm{mol} / \mathrm{L}$, which indicated that honokiol activated SIRT1 promoter in HUVECs.

\section{siSIRT1 further overturned the effect of honokiol on SIRT1 expression, cell viability and apoptosis of HG/HF-induced HUVECS}

As shown in Fig. 3A-C, the expression of SIRT1 in the treated cells was detected by Western blot and RTPCR. The data showed that HG/HF obviously inhibited the expression of SIRT1 compared with the siNC group $(p<0.001)$, but significantly promoted the expression of SIRT1 compared with the $\mathrm{HG} / \mathrm{HF}+\operatorname{siNC}$ group $(p<$ 0.001) siSIRT1 sharply inhibited the expression of SIRT1 compared with the HG/HF + honokiol + siNC group $(p<0.001)$. By examining cell viability, we found that HG/HF significantly inhibited cell viability in comparison with the siNC group $(p<0.001)$, while honokiol reversed the inhibitory effect of $\mathrm{HG} / \mathrm{HF}$ on cell viability in comparison with the $\mathrm{HG} / \mathrm{HF}+\operatorname{siNC}$ group $(p<0.01)$. siSIRT1 further overturned the effect of honokiol on cell viability when compared with the HG/HF + honokiol + siNC group (Fig. 3D, $p<0.05$ ). Additionally, the apopto- sis experiment results showed that $\mathrm{HG} / \mathrm{HF}$ evidently promoted apoptosis as compared with the siNC group $(p<$ 0.001), however, honokiol counteracted the effect of $\mathrm{HG} / \mathrm{HF}$ on promoting apoptosis as compared with the $\mathrm{HG} / \mathrm{HF}+\operatorname{siNC}$ group $(p<0.001)$. Moreover, siSIRT1 further reversed the effect of honokiol on cell apoptosis as compared with the $\mathrm{HG} / \mathrm{HF}+$ honokiol + siNC group (Fig. 3E-F, $p<0.001$ ).

\section{siSIRT1 reversed the effect of honokiol on ROS, $M D A$ and SOD contents in $\mathrm{HG} / \mathrm{HF}$-induced HUVECS}

We found that $\mathrm{HG} / \mathrm{HF}$ greatly increased the contents of ROS and MDA as compared with the siNC group ( $p<$ 0.001), however, honokiol inhibited the effect of $\mathrm{HG} / \mathrm{HF}$ on promoting the contents of ROS and MDA in the cells compared with the HG/HF + siNC group $(p<0.01)$, but siSIRT1 further reversed the effect of honokiol as compared with the HG/HF + honokiol + siNC group (Fig. $4 \mathrm{~A}-\mathrm{B}, p<0.05$ ). As shown in Figure 4C, the content of SOD was significantly reduced in $\mathrm{HG} / \mathrm{HF}+$ siNC group compared with the siNC group $(p<0.001)$, but was increased by honokiol compared with the $\mathrm{HG} / \mathrm{HF}+$ siNC group $(p<0.001)$. However, siSIRT1 inhibited the content of SOD compared with the HG/HF + honokiol + siNC group (Fig. $4 \mathrm{C}, p<0.01$ ).

\section{siSIRT1 reversed the effect of honokiol on the expressions of ER stress markers, cleaved caspase-3, and $\mathrm{p}-\mathrm{Akt}$}

Western blot results showed that $\mathrm{HG} / \mathrm{HF}$ promoted the expressions of CHOP, GRP78, p-PERK, p-IRE1 $\alpha$ and cleaved caspase-3 compared with the siNC group $(p<$ 0.001), while honokiol partially counteracted the effect of $\mathrm{HG} / \mathrm{HF}$ on promoting the expression levels of ER markers and cleaved caspase- 3 compared with the $\mathrm{HG} / \mathrm{HF}+\operatorname{siNC}$ group $(p<0.01)$. siSIRT1 further overturned the effect of honokiol on the cells when compared with the $\mathrm{HG} / \mathrm{HF}+$ honokiol + siNC group (Fig. 4D-E, $p$ $<0.05$ ). In addition, the results of Western blot also demonstrated that $\mathrm{HG} / \mathrm{HF}$ inhibited the expression of $\mathrm{p}$-Akt compared with the siNC group $(p<0.01)$, while honokiol reversed the inhibitory effect of $\mathrm{HG} / \mathrm{HF}$ on the expression of p-Akt compared with the $\mathrm{HG} / \mathrm{HF}+$ siNC group $(p<0.05)$. However, siSIRT1 inhibited the promoting effect of Honokiol on the expression of p-Akt compared with the HG/HF + honokiol + siNC group (Fig. 4F-G, $p<0.001$ ). However, Akt expression did not differ among groups. Therefore, the p-Akt/Akt ratio was sharply decreased after $\mathrm{HG} / \mathrm{HF}$ intervention as compared with the siNC group $(p<0.001)$, however, honokiol promoted the p-Akt/Akt ratio as compared with the HG/HF + siNC group $(p<0.01)$. siSIRT1 partially reversed the 


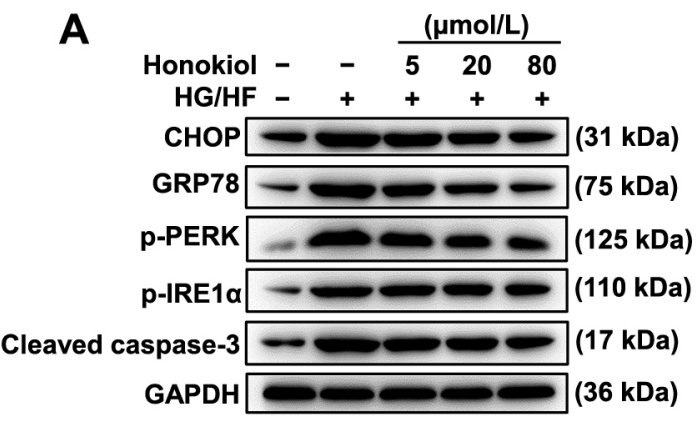

\section{B}

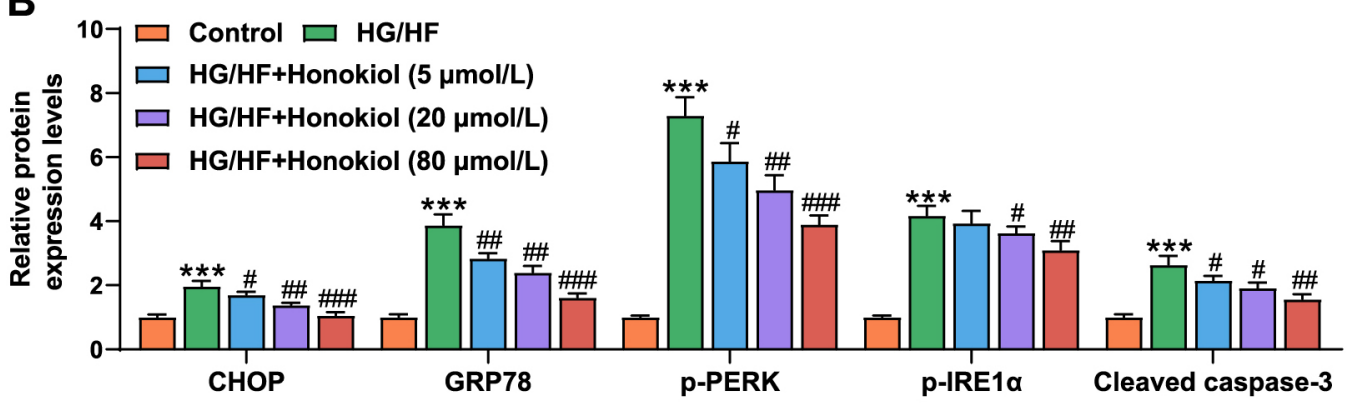

C

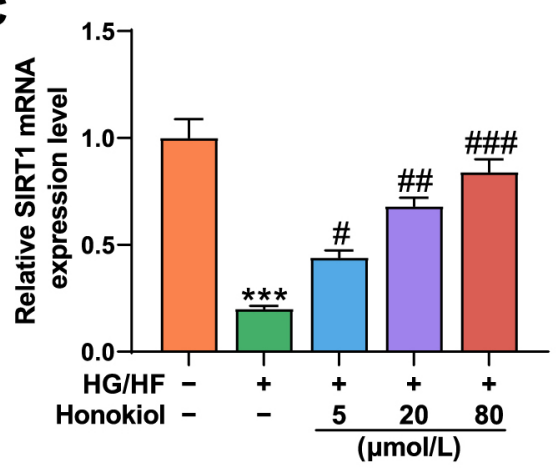

D

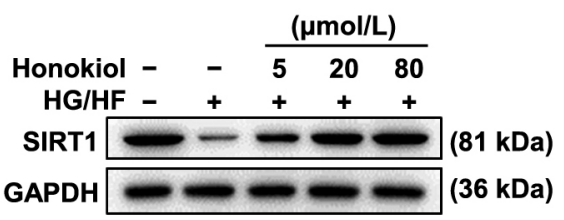

E

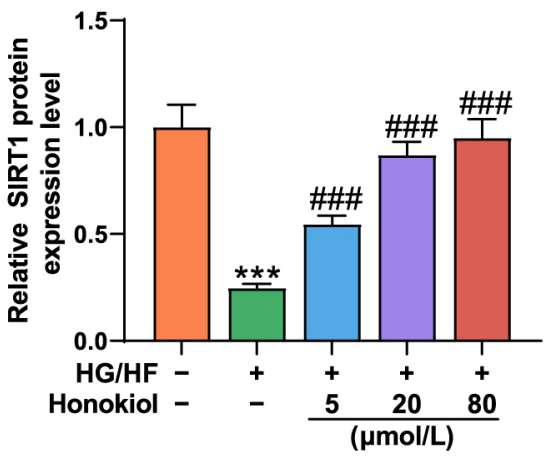

$\mathbf{F}$

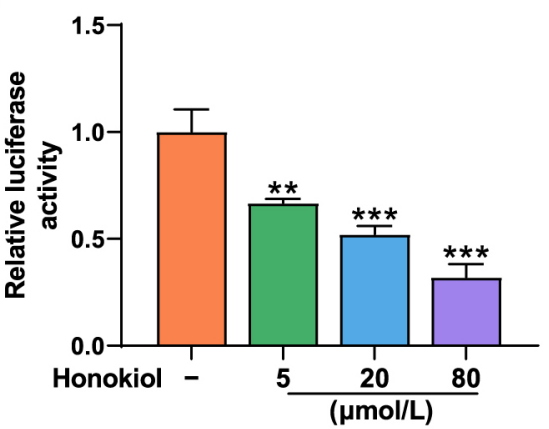

Fig. 2 Honokiol reversed the effect of $\mathrm{HG} / \mathrm{HF}$ on promoting the expression of ER stress markers and cleaved caspase-3 as well as the inhibitory effect on SIRT1 expression in HG/HF-induced HUVECs

(A-B) The expressions of CHOP, GRP78, p-PERK and p-IRE1 $\alpha$ as well as cleaved caspase-3 in the treated cells were examined by Western blot, and GAPDH was used as an internal loading control. (C) The expression of SIRT1 in treated cells was examined by RT-qPCR, and GAPDH was used as a reference gene. (D-E) The expression of SIRT1 in treated cells was examined by Western blot, and GAPDH was used as an internal loading control. (F) The relative luciferase activity of SIRT1 promoter in HUVECs treated with honokiol was detected by dual luciferase reporter analysis. $* * * p<0.001 v$ s. Control; ${ }^{\#} p<0.05,{ }^{\#} p<0.01$, \#\#\# $p<0.001 v s$. HG/HF; ${ }^{* *} p<0.01,{ }^{* * *} p<0.001$ vs. Honokiol (-) $(0 \mu \mathrm{mol} / \mathrm{L}$ of Honokiol). (HG/HF, high glucose/high fat; HUVECs, human umbilical vein endothelial cells; ER stress, endoplasmic reticulum stress; CHOP, C/EBP homologous protein; GRP78, glucose-regulated protein 78; p-PERK, phosphorylated-protein kinase RNA-like endoplasmic reticulum kinase; p-IRE1 $\alpha$, phosphorylated-inositol requiring enzyme-1 $\alpha$; RT-qPCR, quantitative reverse transcription PCR) 


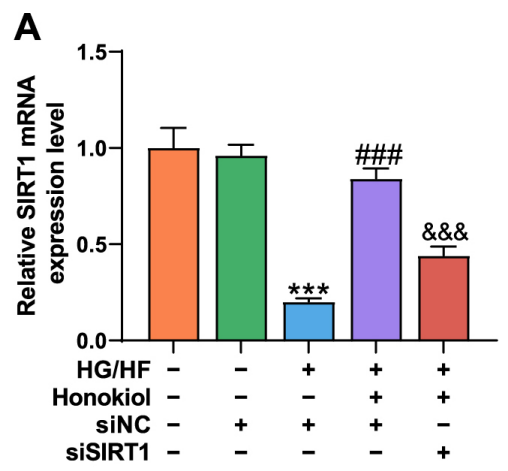

B
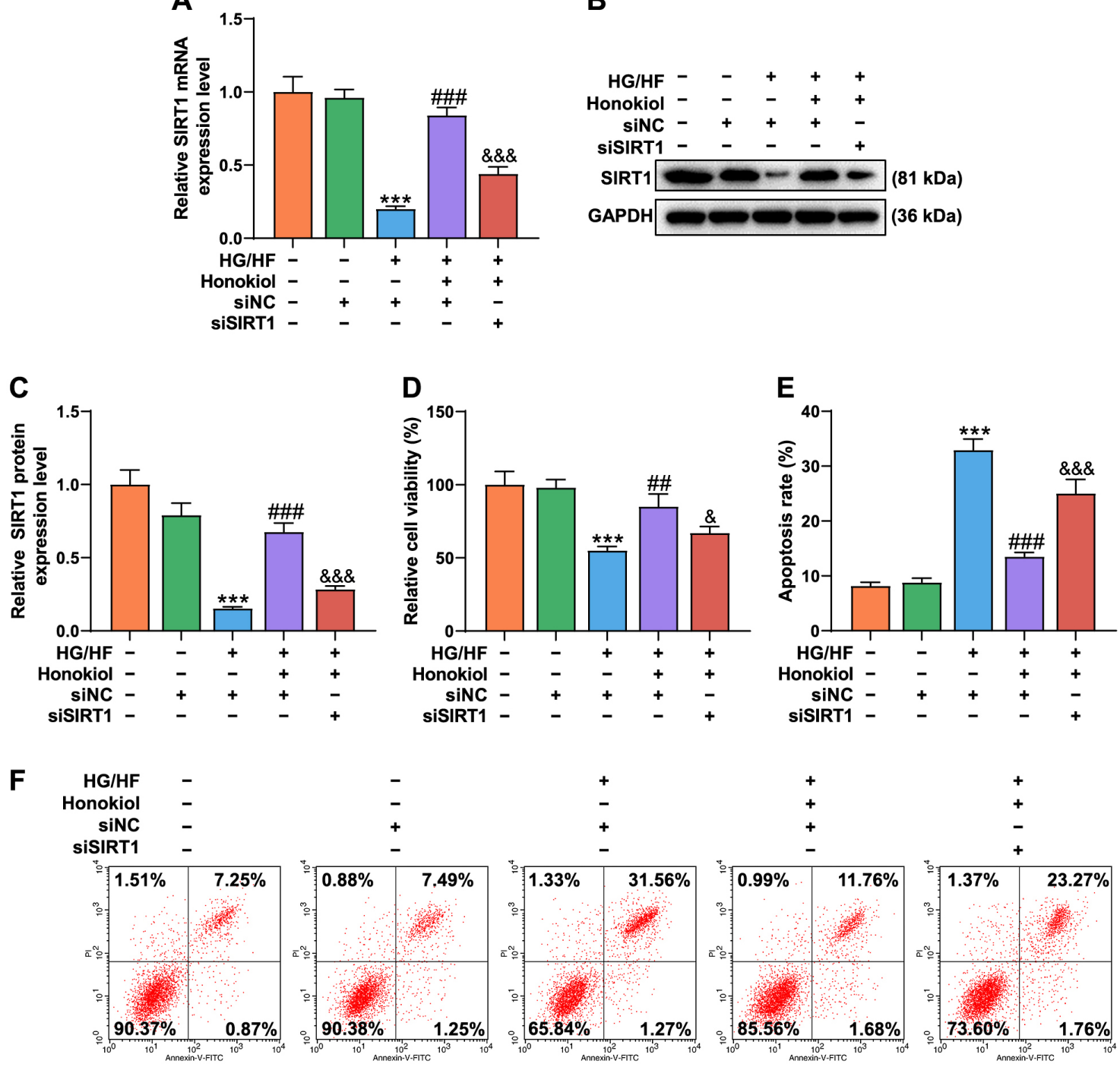

Fig. 3 siSIRT1 further overturned the effect of honokiol on SIRT1 expression, cell viability and apoptosis of HG/HF-induced HUVECs

(A) The expression of SIRT1 in honokiol treatment cells after transfection was examined by RT-qPCR, and GAPDH was used as a reference gene. (B-C) The expression of SIRT1 in honokiol treatment cells after transfection was examined by Western blot, and GAPDH was used as an internal loading control. (D) The viability of honokiol treatment cells after transfection was examined by MTT. (E-F) The apoptosis of honokiol treatment cells after transfection was examined by flow cytometry. *** $p<0.001 v s$. siNC; \# $p<0.01,{ }^{\# \#} p<0.001$ vs. HG/HF + siNC; \& $p<0.05$, \&\&\& $p<0.001$ vs. HG/HF + honokiol + siNC. (HG/HF, high glucose/high fat; HUVECs, human umbilical vein endothelial cells; RT-qPCR, quantitative reverse transcription PCR; MMT, Methyl tetrazolium)

effect of honokiol on the p-Akt/Akt ratio in HUVECs as compared with the $\mathrm{HG} / \mathrm{HF}+$ honokiol + siNC group (Fig. 4H, $p<0.001$ ).

\section{SIRT1 directly modulated activity of Akt in HUVECs}

Furthermore, the direct interrelation between SIRT1 and Akt was detected with Co-IP assay in HUVECs (Fig. $5 \mathrm{~A})$. By the same way, we observed that the acetylation of Akt was increased in HUVECs when SIRT1 was knocked down (Fig. 5B).

\section{Discussion}

Oxidative stress injury and changes in blood glucose and blood lipids are closely related to the development of diabetes, and honokiol has positive effects on antioxidation and regulation of blood glucose and a high safety [20-23]. Exposure of HUVECs to HG/HF could mimic diabetic vascular endothelial cells in a physiological condition [29]. To explore the effect of honokiol on HG/HF-induced diabetic vascular endothelial cells, HUVECs was induced by $\mathrm{HG} / \mathrm{HF}$ following a previous report [27, 28]. The results of related experiments showed that high-glucose treatment inhibited the viabil- 
A

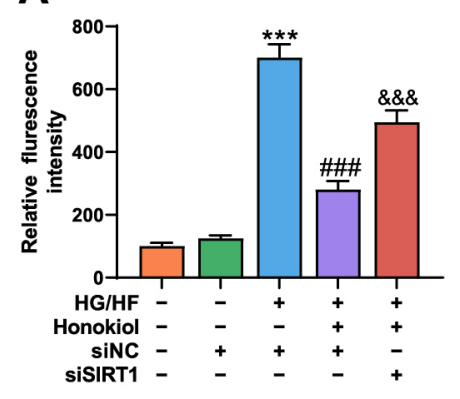

B

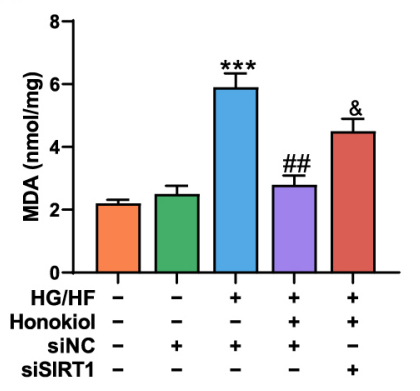

C

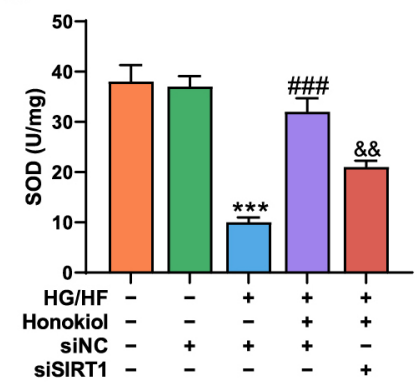

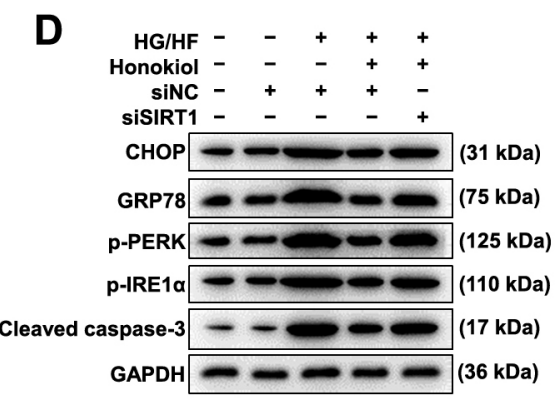

E

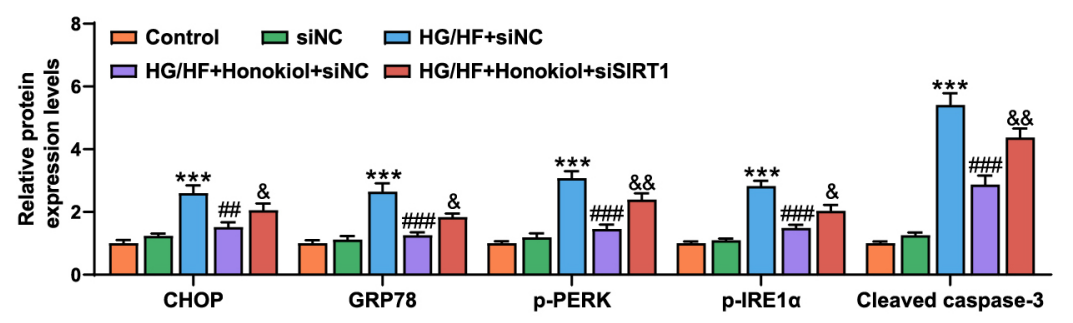

$\mathbf{F}$

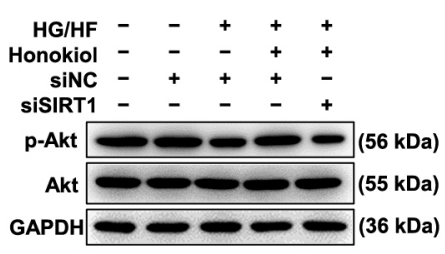

G

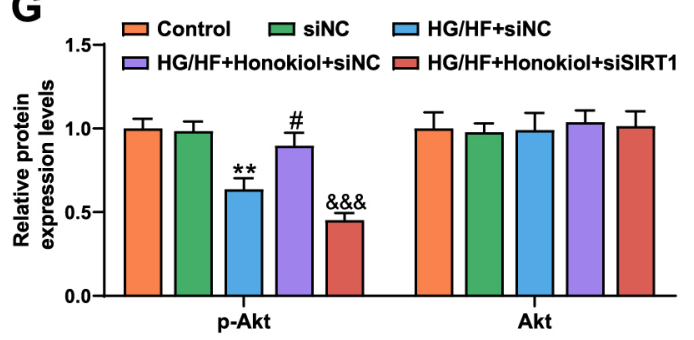

H

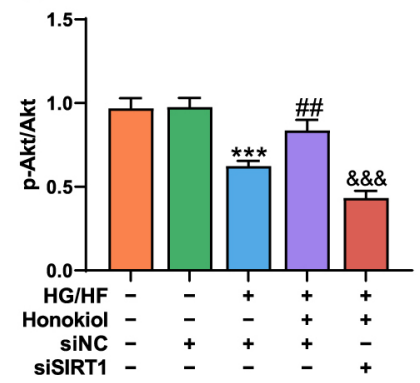

Fig. 4 siSIRT1 reversed the effect of honokiol on the contents of ROS, MDA and SOD, and the expressions of ER stress markers and cleaved caspase-3 as well as the p-Akt (A) The relative fluorescence intensity of cells after transfection was examined. (B) The MDA content of cells after transfection was examined. (C) The SOD content of cells after transfection was examined. (D-E) The expressions of CHOP, GRP78, p-PERK and p-IRE1 $\alpha$ as well as cleaved caspase-3 in transfected cells were examined by Western blot, and GAPDH was used as an internal loading control. (F-G) The expressions of p-Akt and Akt in transfected cells were examined by Western blot, and GAPDH was used as an internal loading control. (H) The ratio of p-Akt to Akt in transfected cells was analyzed. $* * p<0.01, * * * p<0.001 v s$. siNC; ${ }^{\#} p<0.05,{ }^{\#} p<0.01,{ }^{\# \#} p<0.001 v s$. HG/HF $+\operatorname{siNC} ;{ }^{*} p<0.05$, , \& $p<0.01$, \&\&\& $p<0.001$ vs. HG/HF + Honokiol + siNC. (ROS, reactive oxygen species; MDA, malondialdehyde; SOD, superoxide dismutase; ER stress, endoplasmic reticulum stress; CHOP, C/EBP homologous protein; GRP78, glucose-regulated protein 78; pPERK, phosphorylated-protein kinase RNA-like endoplasmic reticulum kinase; p-IRE1 $\alpha$, phosphorylated-inositol requiring enzyme-1 $\alpha$; p-Akt, phosphorylated-Akt)

ity of the HUVECs and promoted apoptosis [30, 31]. In this experiment, we also found that $\mathrm{HG} / \mathrm{HF}$ significantly inhibited viability and promoted apoptosis of HUVECs, but the effect of HG/HF on HUVECs should be further validated.

ROS are highly reactive small molecules that can oxidize lipids and proteins, and play an important role in cell signaling and homeostasis. ROS levels will significantly increase under abnormal environments, and high glucose can induce ROS production $[32,33]$. MDA is a stable product of lipid peroxidation, and the level of MDA is generally considered as a marker of oxidative stress, and high glucose can also induce an increase in MDA content [34-36]. The SOD family plays an important role in the regulation of oxidative stress, and the activity of SOD is decreased in a high-glucose environment $[37,38]$. In this experiment, we found that $\mathrm{HG} / \mathrm{HF}$ increased the levels of ROS and MDA and reduced the level of SOD, but honokiol reversed the effect of $\mathrm{HG} / \mathrm{HF}$ on cells. Moreover, the experimental results showed that honokiol could reduce ER stress, but this should be further verified at a molecular level.

Studies have shown that CHOP is involved in ER stress-mediated apoptosis pathway, and excessive ER 
A

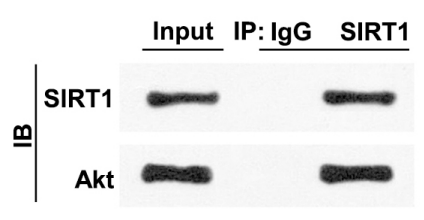

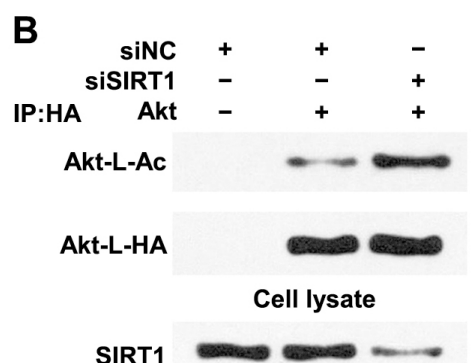

Fig. 5 SIRT1 directly modulated activity of Akt in HUVECs

(A) Co-IP technique was performed to investigate the interrelation between SIRT1 and Akt in HUVECs. (B) Deacetylation of Akt by SIRT1 was detected by Co-IP assay after overexpressing Akt and knocking down SIRT1 in HUVECs. Co-IP, Coimmunoprecipitation; L-Ac, lysine acetylation.

stress can lead to CHOP-mediated apoptosis [39, 40]. In addition, it has been found that GRP78 is a heat-shock protein and a key regulator of ER stress, similarly, both the PERK pathway and IRE1 $\alpha$ are important regulators of ER stress response [41-43]. In the process of apoptosis, caspase is responsible for the majority of proteolysis, therefore the detection of the expression level of cleaved caspase-3 is considered as a marker of apoptosis [44]. When stimulated by external stimuli, cells activate ER stress and further activate signaling pathways, thereby inducing cell death and apoptosis [45]. Moreover, honokiol has been found to regulate cell migration and apoptosis in human lung cancer cells by regulating the expression of ER stress-induced apoptotic signaling molecules, such as CHOP, GRP78, p-PERK and Cleaved caspase 9 [46]. In this experiment, we found that $\mathrm{HG} / \mathrm{HF}$ promoted the expressions of ER markers and cleaved caspase-3, however, Honokiol reversed the effects of HG/HF on ER markers and cleaved caspase-3 in cells. Studies indicated that increased ROS generation and ER stress will interact with each other [47, 48]. The above experimental results illustrated that honokiol reversed HG/HF-induced decreases of viability and apoptosis by inhibiting ER stress response. However, the mechanism of action of honokiol remained unclear.

SIRT1 belongs to the Sirtuins family, and recent studies have shown that SIRT1 plays an important role in inflammation, oxidative stress and ER stress [49-51]. SIRT1 was found to inhibit ER stress-induced apoptosis in mouse experiments [51]. Losartan inhibited ER stress of tubular epithelial cells by up-regulating SIRT1 [52]. In addition, honokiol could reduce oxidative stress and apoptosis in rats through mediating the SIRT1-Nrf2 signaling pathway [26]. Resveratrol suppresses apoptosis of rat hippocampal cells through inhibiting ER stress and activating the SIRT1/AMPK/Akt signaling pathway, and the PI3K-Akt-GSK3 $\beta$ signaling pathway is involved in the effect of SIRT1 on ER stress $[19,53]$. We found that
HG/HF inhibited the expression of SIRT1, and that siSIRT1 reversed the effect of honokiol on the expressions of ER stress markers and cleaved caspase- 3 as well as the p-Akt.

Avtanski et al. [54] found that SIRT1 is involved in the beneficial effects of honokiol in antagonizing the oncogenic actions of leptin in breast cancer. Zhang et al. [54] found that honokiol relieves myocardial ischemia/ reperfusion injury in Type 1 diabetic rats by inhibiting oxidative stress and apoptosis via activating the SIRT1Nrf2 signaling pathway. These findings did not reveal whether honokiol can activate SIRT1 promoter or not, but they only demonstrated that honokiol increased SIRT1 expression or activity. In our study, we found that honokiol increased SIRT1 expression and we further confirmed that honokiol activated SIRT1 promoter. Moreover, SIRT1 directly interacted with Akt, consequently regulating Akt activity. Collectively, these data indicated that honokiol regulates SIRT1 expression thereby controlling the Akt pathway regulating ER stress of HG/HF-induced HUVECs.

This study has several limitations. Although the present study have reported protective effect and potential mechanism of honokiol on HG/HF-induced HUVECs, further experiments are needed to perform on animal model.

In conclusion, our results indicate that honokiol directly regulates SIRT1 expression to modulate ER stress through activating the Akt pathway, and this in turn promotes cell viability and inhibits apoptosis of $\mathrm{HG} /$ HF-induced HUVECs. Our results provide a theoretical basis for honokiol as a new drug for the treatment of patients with diabetic vascular complications.

\section{Funding}

Not applicable. 


\section{Competing Interests}

The authors declare no conflicts of interest.

\section{Acknowledgements}

Not applicable.

\section{Reference}

1. Woldaregay AZ, Arsand E, Botsis T, Albers D, Mamykina L, et al. (2019) Data-driven blood glucose pattern classification and anomalies detection: machine-learning applications in type 1 diabetes. $J$ Med Internet Res 21: e11030.

2. Wang J, Ma Q, Li Y, Li P, Wang M, et al. (2020) Research progress on Traditional Chinese Medicine syndromes of diabetes mellitus. Biomed Pharmacother 121: 109565.

3. Gutierrez-Rodelo C, Roura-Guiberna A, Olivares-Reyes JA (2017) Molecular mechanisms of insulin resistance: an update. Gac Med Mex 153: 214-228 (In Spanish).

4. Forbes JM, Cooper ME (2013) Mechanisms of diabetic complications. Physiol Rev 93: 137-188.

5. Lombardi R, Airaghi L, Targher G, Serviddio G, Maffi G, et al. (2020) Liver fibrosis by FibroScan ${ }^{\circledR}$ independently of established cardiovascular risk parameters associates with macrovascular and microvascular complications in patients with type 2 diabetes. Liver Int 40: 347-354.

6. Vijan S (2019) Type 2 diabetes. Ann Intern Med 171: Itc65-itc80.

7. Kim C, Kim B (2018) Anti-cancer natural products and their bioactive compounds inducing ER stress-mediated apoptosis: a review. Nutrients 10: 1021.

8. Dunlop RA, Powell JT, Metcalf JS, Guillemin GJ, Cox PA (2018) L-serine-mediated neuroprotection includes the upregulation of the ER stress chaperone protein disulfide isomerase (PDI). Neurotox Res 33: 113-122.

9. Chipurupalli S, Kannan E, Tergaonkar V, D'Andrea R, Robinson N (2019) Hypoxia induced ER stress response as an adaptive mechanism in cancer. Int J Mol Sci 20: 749.

10. Jia LX, Zhang WM, Li TT, Liu Y, Piao CM, et al. (2017) ER stress dependent microparticles derived from smooth muscle cells promote endothelial dysfunction during thoracic aortic aneurysm and dissection. Clin Sci (Lond) 131: 1287-1299.

11. Battson ML, Lee DM, Gentile CL (2017) Endoplasmic reticulum stress and the development of endothelial dysfunction. Am J Physiol Heart Circ Physiol 312: H355H367.

12. Dandekar A, Mendez R, Zhang K (2015) Cross talk between ER stress, oxidative stress, and inflammation in health and disease. Methods Mol Biol 1292: 205-214.

13. Dong Y, Fernandes C, Liu Y, Wu Y, Wu H, et al. (2017) Role of endoplasmic reticulum stress signalling in diabetic endothelial dysfunction and atherosclerosis. Diab Vasc Dis Res 14: 14-23.

14. Maamoun H, Abdelsalam SS, Zeidan A, Korashy HM, Agouni A (2019) Endoplasmic reticulum stress: a critical molecular driver of endothelial dysfunction and cardiovascular disturbances associated with diabetes. Int $\mathrm{J} \mathrm{Mol} \mathrm{Sci}$
20: 1658

15. Jung TW, Park HS, Jeong JH, Lee T (2019) Salsalate ameliorates the atherosclerotic response through HO-1and SIRT1-mediated suppression of ER stress and inflammation. Inflamm Res 68: 655-663.

16. Osum M, Serakinci N (2020) Impact of circadian disruption on health; SIRT1 and telomeres. DNA Repair (Amst) 96: 102993.

17. Pires Da Silva J, Monceaux K, Guilbert A, Gressette M, Piquereau J, et al. (2020) SIRT1 protects the heart from ER stress-induced injury by promoting eEF2K/eEF2dependent autophagy. Cells 9: 426.

18. Zhang L, Bao D, Li P, Lu Z, Pang L, et al. (2018) Particle-induced SIRT1 downregulation promotes osteoclastogenesis and osteolysis through ER stress regulation. Biomed Pharmacother 104: 300-306.

19. Koga T, Suico MA, Shimasaki S, Watanabe E, Kai Y, et al. (2015) Endoplasmic reticulum (ER) stress induces sirtuin 1 (SIRT1) expression via the PI3K-Akt-GSK3beta signaling pathway and promotes hepatocellular injury. $J$ Biol Chem 290: 30366-30374.

20. Sarrica A, Kirika N, Romeo M, Salmona M, Diomede L (2018) Safety and toxicology of magnolol and honokiol. Planta Med 84: 1151-1164.

21. Jeong YH, Hur HJ, Jeon EJ, Park SJ, Hwang JT, et al. (2018) Honokiol improves liver steatosis in ovariectomized mice. Molecules 23: 194.

22. Dai X, Li RZ, Jiang ZB, Wei CL, Luo LX, et al. (2018) Honokiol inhibits proliferation, invasion and induces apoptosis through targeting lyn kinase in human lung adenocarcinoma cells. Front Pharmacol 9: 558.

23. Seo MS, Kim JH, Kim HJ, Chang KC, Park SW (2015) Honokiol activates the LKB1-AMPK signaling pathway and attenuates the lipid accumulation in hepatocytes. Toxicol Appl Pharmacol 284: 113-124.

24. Kim YJ, Choi MS, Cha BY, Woo JT, Park YB, et al. (2013) Long-term supplementation of honokiol and magnolol ameliorates body fat accumulation, insulin resistance, and adipose inflammation in high-fat fed mice. Mol Nutr Food Res 57: 1988-1998.

25. Choi SS, Cha BY, Lee YS, Yonezawa T, Teruya T, et al. (2012) Honokiol and magnolol stimulate glucose uptake by activating PI3K-dependent Akt in L6 myotubes. Biofactors 38: 372-377.

26. Zhang B, Zhai M, Li B, Liu Z, Li K, et al. (2018) Honokiol ameliorates myocardial ischemia/reperfusion injury in type 1 diabetic rats by reducing oxidative stress and apoptosis through activating the SIRT1-Nrf2 signaling pathway. Oxid Med Cell Longev 2018: 3159801.

27. Zhu D, Wang H, Zhang J, Zhang X, Xin C, et al. (2015) 
Irisin improves endothelial function in type 2 diabetes through reducing oxidative/nitrative stresses. $J$ Mol Cell Cardiol 87: 138-147.

28. Tan Z, Liu H, Song X, Ling Y, He S, et al. (2019) Honokiol post-treatment ameliorates myocardial ischemia/ reperfusion injury by enhancing autophagic flux and reducing intracellular ROS production. Chem Biol Interact 307: 82-90.

29. Cao X, Han C, Wen J, Guo X, Zhang K (2018) Nicotine increases apoptosis in HUVECs cultured in high glucose/ high fat via Akt ubiquitination and degradation. Clin Exp Pharmacol Physiol 45: 198-204.

30. Jiang T, Jiang D, Zhang L, Ding M, Zhou H (2019) Anagliptin ameliorates high glucose- induced endothelial dysfunction via suppression of NLRP3 inflammasome activation mediated by SIRT1. Mol Immunol 107: 54-60.

31. Zhang J, Guo Y, Ge W, Zhou X, Pan M (2018) High glucose induces the apoptosis of HUVECs in mitochondria dependent manner by enhancing VDAC1 expression. Pharmazie 73: 725-728.

32. Scherz-Shouval R, Elazar Z (2011) Regulation of autophagy by ROS: physiology and pathology. Trends Biochem Sci 36: 30-38.

33. Luo J, Xiang Y, Xu X, Fang D, Li D, et al. (2018) High glucose-induced ROS production stimulates proliferation of pancreatic cancer via inactivating the JNK pathway. Oxid Med Cell Longev 2018: 6917206.

34. Gawel S, Wardas M, Niedworok E, Wardas P (2004) Malondialdehyde (MDA) as a lipid peroxidation marker. Wiad Lek 57: 453-455 (In Polish).

35. Domijan AM, Ralic J, Radic Brkanac S, Rumora L, ZanicGrubisic T (2015) Quantification of malondialdehyde by HPLC-FL-application to various biological samples. Biomed Chromatogr 29: 41-46.

36. Zhang W, Song J, Zhang Y, Ma Y, Yang J, et al. (2018) Intermittent high glucose-induced oxidative stress modulates retinal pigmented epithelial cell autophagy and promotes cell survival via increased HMGB1. BMC Ophthalmol 18: 192.

37. Zheng DH, Han ZQ, Wang XX, Ma D, Zhang J (2019) Erythropoietin attenuates high glucose-induced oxidative stress and inhibition of osteogenic differentiation in periodontal ligament stem cell (PDLSCs). Chem Biol Interact 305: 40-47.

38. Bresciani G, da Cruz IB, Gonzalez-Gallego J (2015) Manganese superoxide dismutase and oxidative stress modulation. Adv Clin Chem 68: 87-130.

39. Oyadomari S, Mori M (2004) Roles of CHOP/GADD153 in endoplasmic reticulum stress. Cell Death Differ 11: 381-389.

40. Liu LQ, Fan ZQ, Tang YF, Ke ZJ (2014) The resveratrol attenuates ethanol-induced hepatocyte apoptosis via inhibiting ER-related caspase-12 activation and PDE activity in vitro. Alcohol Clin Exp Res 38: 683-693.
41. Chou CK, Liu W, Hong YJ, Dahms HU, Chiu CH, et al. (2018) Ethyl acetate extract of scindapsus cf. hederaceus exerts the inhibitory bioactivity on human non-small cell lung cancer cells through modulating ER stress. Int $J$ Mol Sci 19: 1832.

42. Dadey DYA, Kapoor V, Khudanyan A, Thotala D, Hallahan DE (2018) PERK regulates glioblastoma sensitivity to ER stress although promoting radiation resistance. Mol Cancer Res 16: 1447-1453.

43. Ibrahim IM, Abdelmalek DH, Elfiky AA (2019) GRP78: a cell's response to stress. Life Sci 226: 156-163.

44. Crowley LC, Waterhouse NJ (2016) Detecting cleaved caspase- 3 in apoptotic cells by flow cytometry. Cold Spring Harb Protoc 2016.

45. Joyce MA, Walters KA, Lamb SE, Yeh MM, Zhu LF, et al. (2009) HCV induces oxidative and ER stress, and sensitizes infected cells to apoptosis in SCID/Alb-uPA mice. PLoS Pathog 5: e1000291.

46. Zhu J, Xu S, Gao W, Feng J, Zhao G (2019) Honokiol induces endoplasmic reticulum stress-mediated apoptosis in human lung cancer cells. Life Sci 221: 204-211.

47. Park IJ, Yang WK, Nam SH, Hong J, Yang KR, et al. (2014) Cryptotanshinone induces G1 cell cycle arrest and autophagic cell death by activating the AMP-activated protein kinase signal pathway in HepG2 hepatoma. Apoptosis 19: 615-628.

48. Malhotra JD, Kaufman RJ (2007) Endoplasmic reticulum stress and oxidative stress: a vicious cycle or a doubleedged sword? Antioxid Redox Signal 9: 2277-2293.

49. Kauppinen A, Suuronen T, Ojala J, Kaarniranta K, Salminen A (2013) Antagonistic crosstalk between NFkappaB and SIRT1 in the regulation of inflammation and metabolic disorders. Cell Signal 25: 1939-1948.

50. Zhang W, Huang Q, Zeng Z, Wu J, Zhang Y, et al. (2017) Sirt1 inhibits oxidative stress in vascular endothelial cells. Oxid Med Cell Longev 2017: 7543973.

51. Liu Z, Gu H, Gan L, Xu Y, Feng F, et al. (2017) Reducing Smad3/ATF4 was essential for Sirt1 inhibiting ER stressinduced apoptosis in mice brown adipose tissue. Oncotarget 8: 9267-9279.

52. Kim H, Baek CH, Lee RB, Chang JW, Yang WS, et al. (2017) Anti-fibrotic effect of losartan, an angiotensin II receptor blocker, is mediated through inhibition of ER stress via up-regulation of SIRT1, followed by induction of HO-1 and thioredoxin. Int J Mol Sci 18: 305.

53. Shati AA (2019) Resveratrol protects against cadmium chloride-induced hippocampal neurotoxicity by inhibiting ER stress and GAAD 153 and activating sirtuin 1/AMPK/ Akt. Environ Toxicol 34: 1340-1353.

54. Avtanski DB, Nagalingam A, Bonner MY, Arbiser JL, Saxena NK, et al. (2015) Honokiol activates LKB1miR-34a axis and antagonizes the oncogenic actions of leptin in breast cancer. Oncotarget 6: 29947-29962. 